\title{
Exploring Chemical and Biological Space of Terpenoids
}

Tao Zeng ${ }^{1, \#}$, Zhihong Liu ${ }^{2, \#}$, Huawei Liu ${ }^{1}$, Wengan $\mathrm{He}^{1}$, Xiaowen Tang ${ }^{1}$, Liwei Xie ${ }^{2}$, Ruibo $\mathrm{Wu}^{1, *}$

${ }^{1}$ School of Pharmaceutical Sciences, Sun Yat-sen University, Guangzhou 510006, P.R. China

${ }^{2}$ State Key Laboratory of Applied Microbiology Southern China, Guangdong Provincial Key Laboratory of Microbial Culture Collection and Application, Guangdong Open Laboratory of Applied Microbiology, Guangdong Institute of Microbiology, Guangdong Academy of Sciences, Guangzhou 510070, China

${ }^{\#}$ T. Zeng and Z. Liu contributed equally to this work.

*E-mail: wurb3@mail.sysu.edu.cn

Table S1 and Figure S1 S7 
Table S1. Terpenoid-like drugs in the DrugBank. ${ }^{a}$

\begin{tabular}{|c|c|c|c|}
\hline $\begin{array}{c}\text { DrugBank } \\
\text { ID } \\
\end{array}$ & Name & Indications & Category \\
\hline DB00137 & Lutein & $\begin{array}{l}\text { Dietary shortage or } \\
\text { imbalance }\end{array}$ & $\mathrm{C} 40$ \\
\hline DB00162 & Vitamin A & $\begin{array}{l}\text { Vitamin A } \\
\text { deficiency }\end{array}$ & $\mathrm{C} 20$ \\
\hline DB00470 & Dronabinol & $\begin{array}{l}\text { Anorexia, Nausea, } \\
\text { Vomiting }\end{array}$ & other \\
\hline DB00825 & Levomenthol & $\begin{array}{l}\text { Pain, Cough, Sore } \\
\text { throat }\end{array}$ & $\mathrm{C} 10$ \\
\hline DB01229 & Paclitaxel & $\begin{array}{l}\text { Kaposi's sarcoma, } \\
\text { Cancer }\end{array}$ & $\mathrm{C} 20$ \\
\hline DB01744 & Camphor & Infection & $\mathrm{C} 10$ \\
\hline DB01780 & Fusicoccin & 1 & $\mathrm{C} 20$ \\
\hline DB02253 & $\begin{array}{l}(1 \mathrm{r})-4- \\
{[(1 \mathrm{e}, 3 \mathrm{e}, 5 \mathrm{e}, 7 \mathrm{z}, 9 \mathrm{e}, 11 \mathrm{z}, 13 \mathrm{e}, 15 \mathrm{e})-17-} \\
\text { Hydroxy-3,7,12,16- } \\
\text { Tetramethylheptadeca- } \\
\text { 1,3,5,7,9,11,13,15-Octaen-1-Y1]- } \\
\text { 3,5,5-Trimethylcyclohex-3-En-1- } \\
\text { O1 }\end{array}$ & l & $\mathrm{C} 30$ \\
\hline DB02296 & Isoamyl alcohol & 1 & $\mathrm{C} 5$ \\
\hline DB02513 & Thymol & Infection & $\mathrm{C} 10$ \\
\hline DB02587 & Colforsin & Hypertension & $\mathrm{C} 20$ \\
\hline DB02914 & $\begin{array}{l}\text { (6e)-6-[(2e,4e,6e)-3,7- } \\
\text { Dimethylnona-2,4,6,8- } \\
\text { Tetraenylidene]-1,5,5- } \\
\text { Trimethylcyclohexene }\end{array}$ & l & $\mathrm{C} 15$ \\
\hline DB02924 & 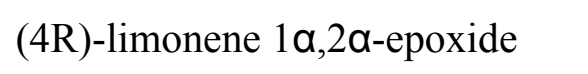 & / & $\mathrm{C} 10$ \\
\hline DB03001 & Peridinin & / & $\mathrm{C} 40$ \\
\hline DB03460 & Violaxanthin & 1 & $\mathrm{C} 40$ \\
\hline DB03750 & Isovaleric Acid & 1 & $\mathrm{C} 5$ \\
\hline DB03852 & Eucalyptol & Inflammation & $\mathrm{C} 10$ \\
\hline DB03961 & $\begin{array}{l}\text { 2c-Methyl-D-Erythritol 2,4- } \\
\text { Cyclodiphosphate }\end{array}$ & / & $\mathrm{C} 5$ \\
\hline DB04376 & 13-Acetylphorbol & 1 & $\mathrm{C} 20$ \\
\hline DB04377 & Meglutol & 1 & $\mathrm{C} 5$ \\
\hline DB05013 & Ingenol mebutate & Actinic keratosis & $\mathrm{C} 20$ \\
\hline DB05767 & Andrographolide & Ulcerative colitis & $\mathrm{C} 20$ \\
\hline DB05974 & Transcrocetinate & Cancer & $\mathrm{C} 20$ \\
\hline
\end{tabular}




\begin{tabular}{|c|c|c|c|}
\hline DB06515 & Resiniferatoxin & $\begin{array}{l}\text { Interstitial cystitis, } \\
\text { Urinary incontinence }\end{array}$ & $\mathrm{C} 20$ \\
\hline DB06543 & Astaxanthin & $\begin{array}{l}\text { Eye disorders, } \\
\text { Cancer, Asthma }\end{array}$ & $\mathrm{C} 40$ \\
\hline DB06743 & Ginkgolide A & Inflammation & $\mathrm{C} 20$ \\
\hline DB06744 & Ginkgolide A & 1 & $\mathrm{C} 20$ \\
\hline DB06745 & Ginkgolide C & 1 & $\mathrm{C} 20$ \\
\hline DB06746 & Ginkgolide J & 1 & $\mathrm{C} 20$ \\
\hline DB06747 & ginkgolide $\mathrm{M}$ & 1 & $\mathrm{C} 20$ \\
\hline DB06755 & Beta carotene & $\begin{array}{l}\text { Macular } \\
\text { Degeneration }\end{array}$ & $\mathrm{C} 40$ \\
\hline DB06903 & $\begin{array}{l}\text { (1S,3aS,5aR,8aS)-1,7,7-trimethyl- } \\
\text { 1,2,3,3a,5a,6,7,8- } \\
\text { octahydrocyclopenta[c]pentalene- } \\
\text { 4-carboxylic acid }\end{array}$ & / & $\mathrm{C} 15$ \\
\hline DB07814 & Gibberellic acid & 1 & $\mathrm{C} 20$ \\
\hline DB07815 & Gibberellin A4 & 1 & $\mathrm{C} 20$ \\
\hline DB08407 & Platensimycin & DB08407 & other \\
\hline DB08921 & (4R)-limonene & 1 & $\mathrm{C} 10$ \\
\hline DB09061 & Cannabidiol & Spasticity & other \\
\hline DB11176 & Zeaxanthin & 1 & $\mathrm{C} 40$ \\
\hline DB11260 & Diacetyl benzoyl lathyrol & 1 & $\mathrm{C} 20$ \\
\hline DB11288 & Borneol & 1 & $\mathrm{C} 10$ \\
\hline DB11344 & $(+)$-menthol & 1 & $\mathrm{C} 10$ \\
\hline DB11345 & (S)-camphor & Ache,Pain & $\mathrm{C} 10$ \\
\hline DB11755 & Tetrahydrocannabivarin & 1 & other \\
\hline DB11874 & Crocin & $\begin{array}{l}\text { Cardiovascular } \\
\text { disease }\end{array}$ & $\mathrm{C} 20$ \\
\hline DB12025 & Triptolide & $\begin{array}{l}\text { HIV, Crohn's } \\
\text { Disease, Intestinal } \\
\text { Diseases, } \\
\text { Gastrointestinal } \\
\text { Diseases, Digestive } \\
\text { System Diseases } \\
\end{array}$ & $\mathrm{C} 20$ \\
\hline DB12327 & Salvinorin A & 1 & $\mathrm{C} 20$ \\
\hline DB12328 & Cantharidin & Warts & $\mathrm{C} 10$ \\
\hline DB12434 & Steviolbioside & HIV-1 Infection & $\mathrm{C} 20$ \\
\hline DB12480 & Betulinic Acid & $\begin{array}{l}\text { Dysplastic Nevus } \\
\text { Syndrome }\end{array}$ & $\mathrm{C} 30$ \\
\hline DB12622 & Lupeol & Acne & $\mathrm{C} 30$ \\
\hline DB12816 & Terpinen-4-ol & 1 & $\mathrm{C} 10$ \\
\hline DB13044 & Gossypol & Cancer & $\mathrm{C} 15$ \\
\hline DB13063 & Parthenolide & Inflammation & $\mathrm{C} 15$ \\
\hline
\end{tabular}




\begin{tabular}{|c|c|c|c|}
\hline DB13089 & Enoxolone & 1 & C30 \\
\hline DB13132 & Artemisinin & Malaria & $\mathrm{C} 15$ \\
\hline DB13751 & Glycyrrhizic acid & $\begin{array}{l}\text { Inflammation, } \\
\text { Cancer }\end{array}$ & $\mathrm{C} 30$ \\
\hline DB14037 & Madecassic acid & 1 & $\mathrm{C} 20$ \\
\hline DB14050 & Cannabidivarin & Rett Syndrome & other \\
\hline DB14054 & Asiatic acid & 1 & $\mathrm{C} 30$ \\
\hline DB14081 & Asiaticoside & 1 & $\mathrm{C} 20$ \\
\hline DB14094 & Tocopherylquinone & 1 & other \\
\hline DB14156 & Synthetic Camphor & 1 & $\mathrm{C} 10$ \\
\hline DB14555 & Ursadiol & 1 & $\mathrm{C} 30$ \\
\hline DB14736 & Cannabivarin & 1 & other \\
\hline DB14737 & Cannabinol & 1 & other \\
\hline DB00170 & Menadione & $\begin{array}{l}\text { Coagulation } \\
\text { disorders }\end{array}$ & $\mathrm{C} 20$ \\
\hline DB00459 & Acitretin & Psoriasis & $\mathrm{C} 20$ \\
\hline DB00486 & Nabilone & Nausea, Vomiting & other \\
\hline DB00523 & Alitretinoin & Cutaneous lesions & $\mathrm{C} 20$ \\
\hline DB00640 & Adenosine & $\begin{array}{l}\text { cardiovascular } \\
\text { disease }\end{array}$ & other \\
\hline DB00657 & Mecamylamine & Hypertension & $\mathrm{C} 10$ \\
\hline DB00755 & Tretinoin & $\begin{array}{l}\text { Acute promyelocytic } \\
\text { leukemia (APL) }\end{array}$ & $\mathrm{C} 20$ \\
\hline DB00926 & Etretinate & Psoriasis & $\mathrm{C} 20$ \\
\hline DB00982 & Isotretinoin & Acne & $\mathrm{C} 20$ \\
\hline DB01022 & Phylloquinone & $\begin{array}{l}\text { Haemorrhagic } \\
\text { conditions }\end{array}$ & C30 \\
\hline DB01248 & Docetaxel & Cancer & $\mathrm{C} 20$ \\
\hline DB01256 & Retapamulin & Impetigo & $\mathrm{C} 20$ \\
\hline DB01463 & Fencamfamin & Depressive fatigue & $\mathrm{C} 10$ \\
\hline DB01637 & $\begin{array}{l}\text { 3,7,11,15-tetramethyl-hexadecan- } \\
\text { 1-ol }\end{array}$ & l & $\mathrm{C} 20$ \\
\hline DB01785 & Dimethylallyl Diphosphate & 1 & $\mathrm{C} 5$ \\
\hline DB01892 & Hyperforin & 1 & $\mathrm{C} 10$ \\
\hline DB02329 & Carbenoxolone & Digestive tract ulcers & $\mathrm{C} 30$ \\
\hline DB02426 & Carboxyatractyloside & 1 & $\mathrm{C} 20$ \\
\hline DB02509 & Farnesol & 1 & $\mathrm{C} 15$ \\
\hline DB02552 & Geranyl Diphosphate & 1 & $\mathrm{C} 10$ \\
\hline DB02640 & Fumagillin & 1 & $\mathrm{C} 15$ \\
\hline DB02699 & 4-Oxoretinol & 1 & $\mathrm{C} 20$ \\
\hline DB02808 & Trifluorofurnesyl Diphosphate & I & $\mathrm{C} 15$ \\
\hline DB02817 & 5-exo-hydroxycamphor & 1 & $\mathrm{C} 10$ \\
\hline DB02851 & Thiocamphor & 1 & $\mathrm{C} 10$ \\
\hline
\end{tabular}




\begin{tabular}{|c|c|c|c|}
\hline DB02860 & Calyculin A & / & $\mathrm{C} 10$ \\
\hline DB02877 & Arotinoid acid & 1 & other \\
\hline DB02906 & $(2 \mathrm{~s}, 4 \mathrm{~s})$-alpha-campholinic acid & 1 & $\mathrm{C} 10$ \\
\hline DB03402 & $\begin{array}{l}\text { 1,2-Di-1-(3,7,11,15-Tetramethyl- } \\
\text { Hexadecane)-Sn-Glycero-3- } \\
\text { Phosphate }\end{array}$ & / & $\mathrm{C} 20$ \\
\hline DB03540 & Norcamphor & 1 & $\mathrm{C} 10$ \\
\hline DB03616 & Kabiramide C & 1 & $\mathrm{C} 20$ \\
\hline DB03646 & 2,3-di-O-phytanyl-sn-glycerol & / & $\mathrm{C} 20$ \\
\hline DB03696 & Lanosterol & 1 & $\mathrm{C} 30$ \\
\hline DB03850 & Jaspisamide A & 1 & $\mathrm{C} 20$ \\
\hline DB03874 & $\begin{array}{l}(4 \mathrm{e}, 8 \mathrm{e}, 12 \mathrm{z}, 16 \mathrm{z})-\mathrm{N}, \mathrm{N}, 4,8,13,17,21- \\
\text { Heptamethyldocosa-4,8,12,16,20- } \\
\text { Pentaen-1-Amine }\end{array}$ & l & $\mathrm{C} 25$ \\
\hline DB04324 & Ovalicin & 1 & $\mathrm{C} 15$ \\
\hline DB04501 & Camphane & l & $\mathrm{C} 10$ \\
\hline DB04695 & Farnesyl thiopyrophosphate & 1 & $\mathrm{C} 15$ \\
\hline DB04702 & $\begin{array}{l}\mathrm{S}-[(2 \mathrm{e})-3,7-\text { dimethylocta-2,6- } \\
\text { dienyl] } \\
\text { trihydrogenthiodiphosphate }\end{array}$ & / & $\mathrm{C} 10$ \\
\hline DB04714 & Isopentenyl pyrophosphate & 1 & $\mathrm{C} 5$ \\
\hline DB04774 & Reidispongiolide A & 1 & other \\
\hline DB04783 & Sphinxolide B & 1 & other \\
\hline DB04864 & Huperzine A & Alzheimer's disease & $\mathrm{C} 15$ \\
\hline DB04930 & Permethrin & Infection & $\mathrm{C} 10$ \\
\hline DB05048 & Cannabinor & pain & other \\
\hline DB05076 & Fenretinide & $\begin{array}{l}\text { Macular } \\
\text { degeneration }\end{array}$ & $\mathrm{C} 20$ \\
\hline DB05186 & SQ-109 & infection & $\mathrm{C} 10$ \\
\hline DB05263 & Caprospinol & Alzheimer's Disease & $\mathrm{C} 30$ \\
\hline DB05265 & Ecabet & $\begin{array}{l}\text { Reflux oesophagitis, } \\
\text { Peptic ulcer disease }\end{array}$ & $\mathrm{C} 20$ \\
\hline DB05297 & Paclitaxel docosahexaenoic acid & Cancer & $\mathrm{C} 20$ \\
\hline DB05653 & IRX-5183 & Cancer & $\mathrm{C} 15$ \\
\hline DB05786 & Irofulven & Cancer & $\mathrm{C} 15$ \\
\hline DB05842 & AI-850 & Cancer & $\mathrm{C} 20$ \\
\hline DB05983 & Bardoxolone methyl & Cancer & $\mathrm{C} 30$ \\
\hline DB06171 & Paclitaxel trevatide & cancer & $\mathrm{C} 20$ \\
\hline DB06347 & Cenisertib & Cancer & $\mathrm{C} 10$ \\
\hline DB06442 & Avasimibe & $\begin{array}{l}\text { cardiovascular } \\
\text { disease }\end{array}$ & $\mathrm{C} 10$ \\
\hline DB06444 & Dexanabinol & Neurologic disorders & other \\
\hline DB06468 & Cariporide & cardiovascular & $\mathrm{C} 10$ \\
\hline
\end{tabular}




\begin{tabular}{|c|c|c|c|}
\hline & & disease & \\
\hline DB06471 & Naxifylline & $\begin{array}{l}\text { cardiovascular } \\
\text { disease }\end{array}$ & $\mathrm{C} 10$ \\
\hline DB06512 & Deramciclane & Anxiety disorders & $\mathrm{C} 10$ \\
\hline DB06581 & Bevirimat & HIV infection & $\mathrm{C} 30$ \\
\hline DB06697 & Artemether & Malaria & $\mathrm{C} 20$ \\
\hline DB06748 & Ginsenoside $\mathrm{C}$ & 1 & $\mathrm{C} 30$ \\
\hline DB06749 & Ginsenoside $\mathrm{Rb} 1$ & 1 & $\mathrm{C} 30$ \\
\hline DB06750 & Ginsenoside Rg1 & 1 & $\mathrm{C} 30$ \\
\hline DB06772 & Cabazitaxel & Cancer & $\mathrm{C} 20$ \\
\hline DB06884 & $\begin{array}{l}\text { 4-hydroxy-N'-(4- } \\
\text { isopropylbenzyl)benzohydrazide }\end{array}$ & / & $\mathrm{C} 10$ \\
\hline DB07031 & $\begin{array}{l}\text { 3-Fluoro-4-\{[(2R)-2-hydroxy-2- } \\
\text { (5,5,8,8-tetramethyl-5,6,7,8- } \\
\text { tetrahydro-2- } \\
\text { naphthalenyl)acetyl]amino }\} \text { benzoi } \\
\text { c acid }\end{array}$ & / & other \\
\hline DB07780 & Farnesyl diphosphate & 1 & $\mathrm{C} 15$ \\
\hline DB07841 & Geranylgeranyl diphosphate & 1 & $\mathrm{C} 20$ \\
\hline DB07895 & $\begin{array}{l}\text { Alpha- } \\
\text { Hydroxyfarnesylphosphonic acid }\end{array}$ & / & $\mathrm{C} 15$ \\
\hline DB08127 & $\begin{array}{l}\text { 1,3,3-trimethyl-2-[(1E,3E)-3- } \\
\text { methylpenta-1,3-dien-1- } \\
\text { yl]cyclohexene }\end{array}$ & / & $\mathrm{C} 15$ \\
\hline DB08180 & $\begin{array}{l}\text { 2-[methyl-(5-geranyl-4-methyl- } \\
\text { pent-3-enyl)-amino]-ethyl- } \\
\text { diphosphate }\end{array}$ & / & $\mathrm{C} 15$ \\
\hline DB08455 & Ro $12-7310$ & 1 & $\mathrm{C} 20$ \\
\hline DB08610 & $\begin{array}{l}\text { N-(2-aminoethyl)-2-\{3-chloro-4- } \\
\text { [(4-isopropylbenzyl)oxy]phenyl }\} \\
\text { acetamide }\end{array}$ & / & $\mathrm{C} 10$ \\
\hline DB08633 & TNP-470 & HIV Infections & $\mathrm{C} 15$ \\
\hline DB08803 & Tymazoline & Inflammation & $\mathrm{C} 10$ \\
\hline DB08955 & Ibuproxam & Inflammatory & $\mathrm{C} 10$ \\
\hline DB08962 & Glibornuride & 1 & $\mathrm{C} 10$ \\
\hline DB09052 & Blinatumomab & $\begin{array}{l}\text { Acute lymphoblastic } \\
\text { leukemia }\end{array}$ & $\mathrm{C} 10$ \\
\hline DB09274 & Artesunate & Malaria & $\mathrm{C} 15$ \\
\hline DB09401 & Isosorbide & 1 & $\mathrm{C} 15$ \\
\hline DB09534 & Ecamsule & UVA rays & $\mathrm{C} 10$ \\
\hline DB11087 & Pyrethrum extract & Insect pest & $\mathrm{C} 10$ \\
\hline DB11096 & Meradimate & UVA rays & $\mathrm{C} 10$ \\
\hline DB11219 & Enzacamene & UVA rays & $\mathrm{C} 10$ \\
\hline
\end{tabular}




\begin{tabular}{|c|c|c|c|}
\hline DB11231 & Lycopene & 1 & $\mathrm{C} 40$ \\
\hline DB11377 & Bambermycins & 1 & other \\
\hline DB11420 & Squalane & 1 & $\mathrm{C} 30$ \\
\hline DB11423 & Lasalocid & $1 /$ & $\mathrm{C} 20$ \\
\hline DB11432 & Narasin & Infection & $\mathrm{C} 20$ \\
\hline DB11460 & Squalene & Cancer & $\mathrm{C} 30$ \\
\hline DB11468 & Tiamulin & 1 & $\mathrm{C} 20$ \\
\hline DB11495 & (S)-methoprene & 1 & $\mathrm{C} 15$ \\
\hline DB11544 & Salinomycin & 1 & $\mathrm{C} 20$ \\
\hline DB11638 & Artenimol & Infection & $\mathrm{C} 15$ \\
\hline DB11669 & Ortataxel & Cancer & $\mathrm{C} 20$ \\
\hline DB11807 & Dexmecamylamine & 1 & $\mathrm{C} 10$ \\
\hline DB11917 & Vatiquinone & 1 & $\mathrm{C} 20$ \\
\hline DB12019 & Tesetaxel & Cancer & $\mathrm{C} 20$ \\
\hline DB12079 & Gefarnate & $\begin{array}{l}\text { Stomach Ulcer, } \\
\text { Duodenal Ulcer, and } \\
\text { Cardiovascular } \\
\text { Disease }\end{array}$ & other \\
\hline DB12112 & Zuretinol acetate & Eye disorder & $\mathrm{C} 20$ \\
\hline DB12144 & BMS-275183 & 1 & $\mathrm{C} 20$ \\
\hline DB12148 & Menatetrenone & \begin{tabular}{|l|} 
Diabetes, \\
Osteoporosis, \\
Prediabetic State, \\
Hepatocellular \\
Carcinoma \\
\end{tabular} & $\mathrm{C} 20$ \\
\hline DB12193 & Ajulemic acid & \begin{tabular}{|l} 
Cystic Fibrosis, \\
Dermatomyositis, \\
Diffuse Cutaneous \\
Systemic Sclerosis \\
\end{tabular} & other \\
\hline DB12326 & Antroquinonol & 1 & $\mathrm{C} 20$ \\
\hline DB12334 & Milataxel & Mesothelioma & $\mathrm{C} 20$ \\
\hline DB12420 & Diazepinomicin & $\begin{array}{l}\text { Glioblastoma } \\
\text { Multiforme }\end{array}$ & $\mathrm{C} 15$ \\
\hline DB12430 & BC-3781 & Infection & $\mathrm{C} 20$ \\
\hline DB12471 & MK-3118 & Infection & $\mathrm{C} 30$ \\
\hline DB12508 & E7107 & cancer & $\mathrm{C} 20$ \\
\hline DB12513 & Omaveloxolone & Cancer & $\mathrm{C} 30$ \\
\hline DB12614 & Reparixin & Cancer,Diabetes & $\mathrm{C} 10$ \\
\hline DB12619 & BMS-188797 & Cancer & $\mathrm{C} 20$ \\
\hline DB12633 & BMS- 184476 & Cancer & $\mathrm{C} 20$ \\
\hline DB12647 & Tocotrienol & 1 & $\mathrm{C} 20$ \\
\hline DB12671 & Beloranib & Diabetes & $\mathrm{C} 15$ \\
\hline DB12681 & Salirasib & Cancer & $\mathrm{C} 15$ \\
\hline
\end{tabular}




\begin{tabular}{|c|c|c|c|}
\hline DB12825 & Lefamulin & l & $\mathrm{C} 20$ \\
\hline DB12984 & \begin{tabular}{|l|} 
Larotaxel \\
\end{tabular} & Cancer & $\mathrm{C} 20$ \\
\hline DB13075 & Menaquinone 7 & $\begin{array}{l}\text { Vitamin K } \\
\text { deficiency }\end{array}$ & $\mathrm{C} 20$ \\
\hline DB13153 & Levomenol & Inflammation & $\mathrm{C} 15$ \\
\hline DB13163 & Terpin hydrate & $\begin{array}{l}\text { Diseases of the } \\
\text { upper respiratory } \\
\text { tract }\end{array}$ & $\mathrm{C} 10$ \\
\hline DB13315 & Sobrerol & 1 & $\mathrm{C} 10$ \\
\hline DB13329 & Guaiazulen & 1 & $\mathrm{C} 15$ \\
\hline DB13368 & Motretinide & 1 & $\mathrm{C} 20$ \\
\hline DB13391 & Levoverbenone & 1 & $\mathrm{C} 10$ \\
\hline DB13600 & Deltamethrin & 1 & $\mathrm{C} 10$ \\
\hline DB13619 & Bornaprine & 1 & $\mathrm{C} 10$ \\
\hline DB13640 & Acetoxolone & 1 & $\mathrm{C} 30$ \\
\hline DB13714 & Xibornol & 1 & $\mathrm{C} 10$ \\
\hline DB13717 & Phenothrin & 1 & $\mathrm{C} 10$ \\
\hline DB13721 & Cypermethrin & 1 & $\mathrm{C} 10$ \\
\hline DB13746 & Bioallethrin & $\begin{array}{l}\text { Lice and scabies } \\
\text { infestation }\end{array}$ & $\mathrm{C} 10$ \\
\hline DB13752 & Tetramethrin & 1 & $\mathrm{C} 10$ \\
\hline DB13809 & Paclitaxel poliglumex & 1 & $\mathrm{C} 20$ \\
\hline DB13828 & Cyfluthrin & 1 & $\mathrm{C} 10$ \\
\hline DB13851 & Artemotil & Malaria & $\mathrm{C} 15$ \\
\hline DB14011 & Nabiximols & Spasticity & other \\
\hline DB14097 & $\begin{array}{l}\text { 13-cis-12-(3'- } \\
\text { Carboxyphenyl)retinoic acid }\end{array}$ & I & $\mathrm{C} 20$ \\
\hline DB14152 & Ginsenosides & l & $\mathrm{C} 30$ \\
\hline DB14256 & Centella asiatica & 1 & other \\
\hline DB14650 & Menadiol diphosphate & 1 & $\mathrm{C} 20$ \\
\hline DB14665 & Bornyl acetate & 1 & $\mathrm{C} 10$ \\
\hline DB14734 & Cannabigerol & 1 & other \\
\hline DB14735 & Cannabichromene & 1 & other \\
\hline
\end{tabular}

${ }^{a}$ ID with bold: compounds also existed in the terpenoid NPs, / :the information is not available in DrugBank. 


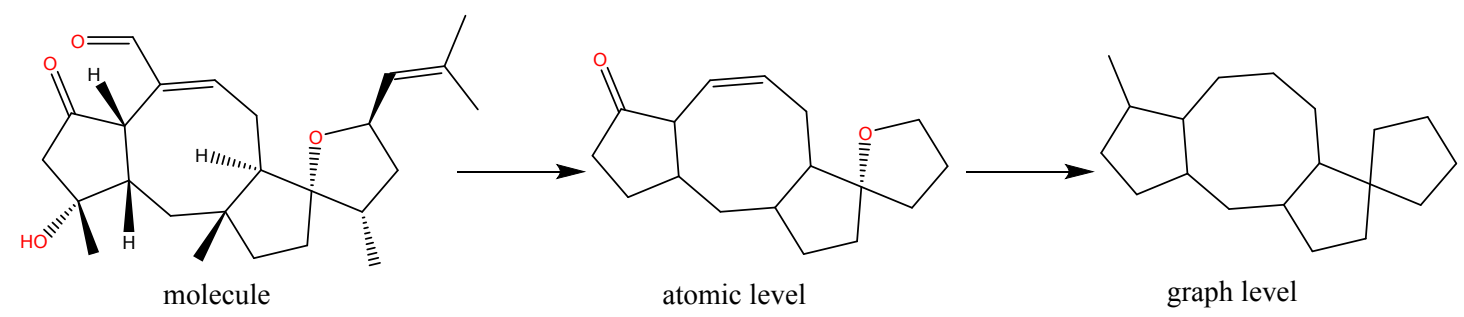

Figure S1. An example of scaffold extraction under Bemis-Murcko framework. The side chains are removed to keep the ring systems and linker. 


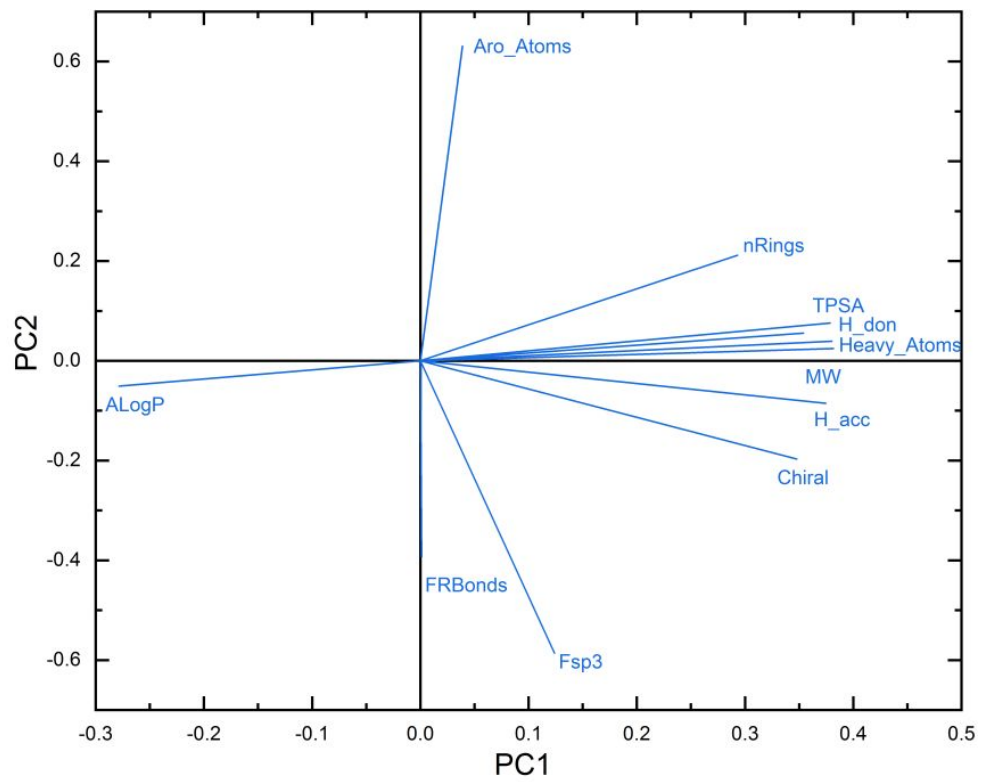

Figure S2. Loading plot for the weight of descriptors in PCA 


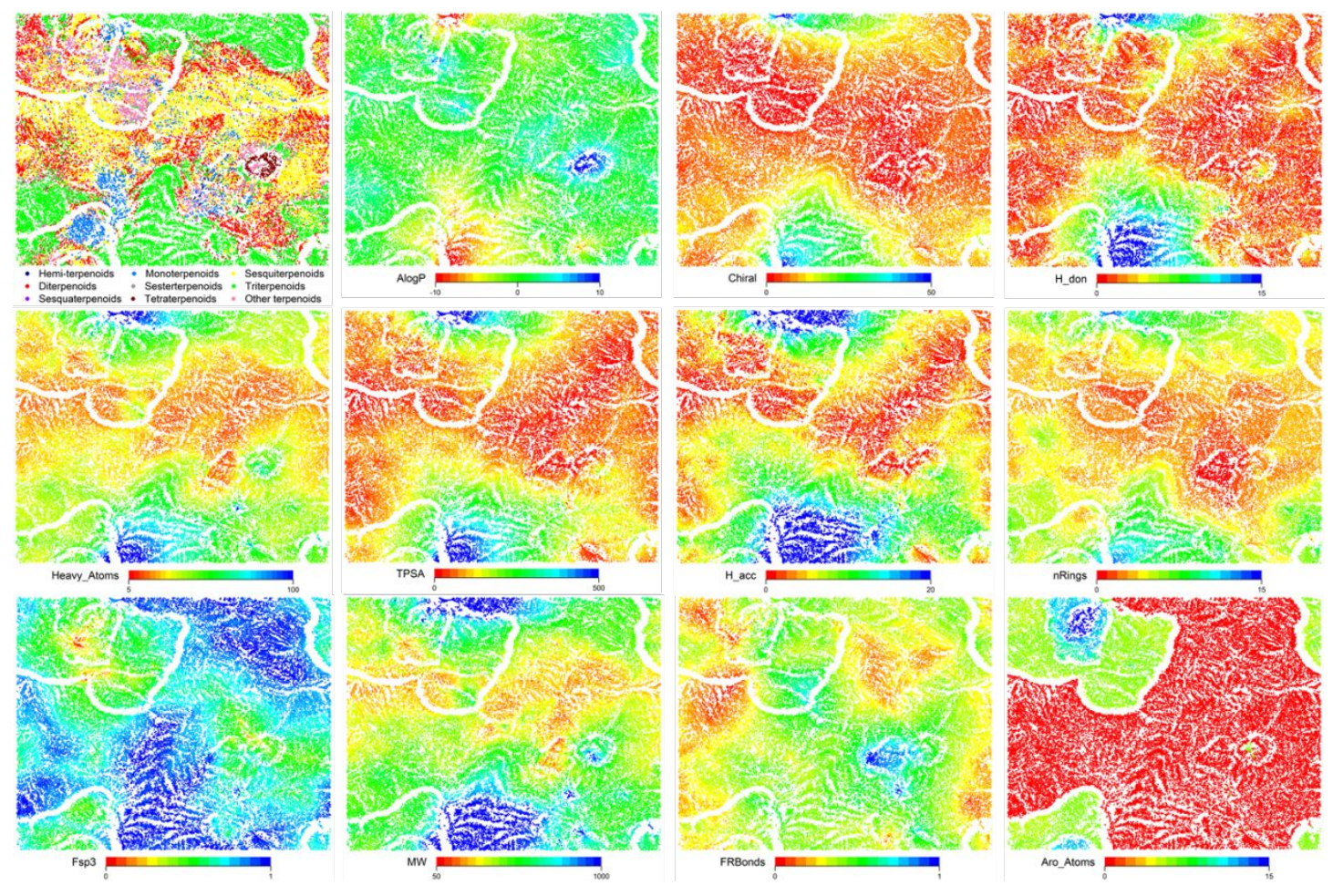

Figure S3. Physicochemical property distribution of terpenoids in SOM mapping. Herein, the peculiarity of triterpenoids is likely due to the saponins-like compound is a major constitute of triterpenoids. The big AlogP values (cyan and blue regions) shown in the second figure is mostly comprised by tetraterpenoids and others $(>C 40)$. The huge areas in red in the last figures indicates the aromatic ring is rare exited in terpenoid NPs. 


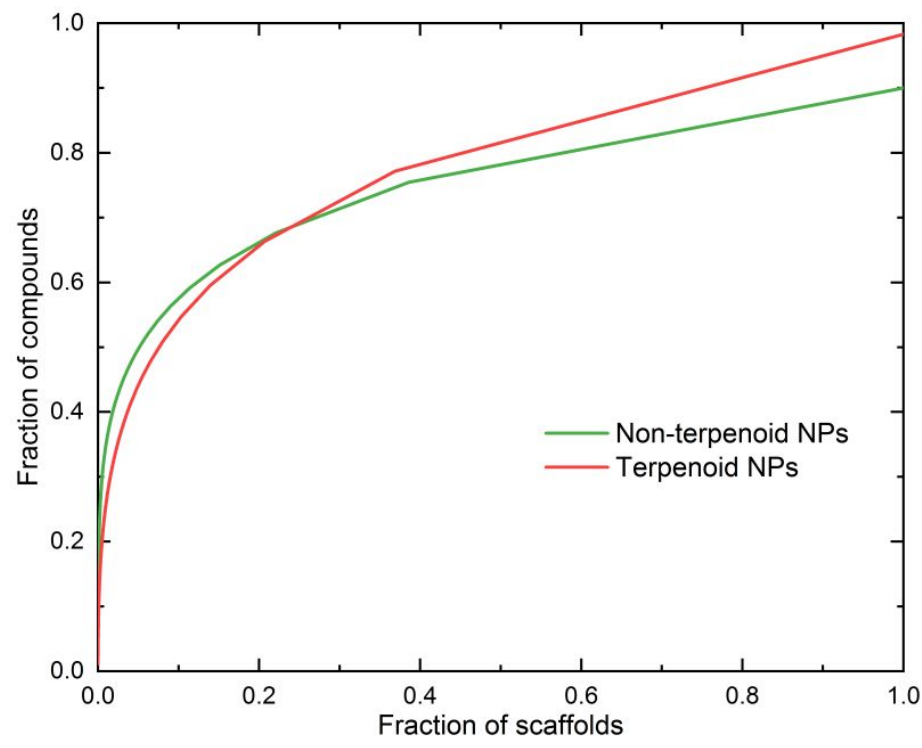

Figure S4. The cyclic system retrieval (CSR) curves for the natural products and terpenoids. The similar curves indicating the close diversity of terpenoid and nonterpenoid NPs 


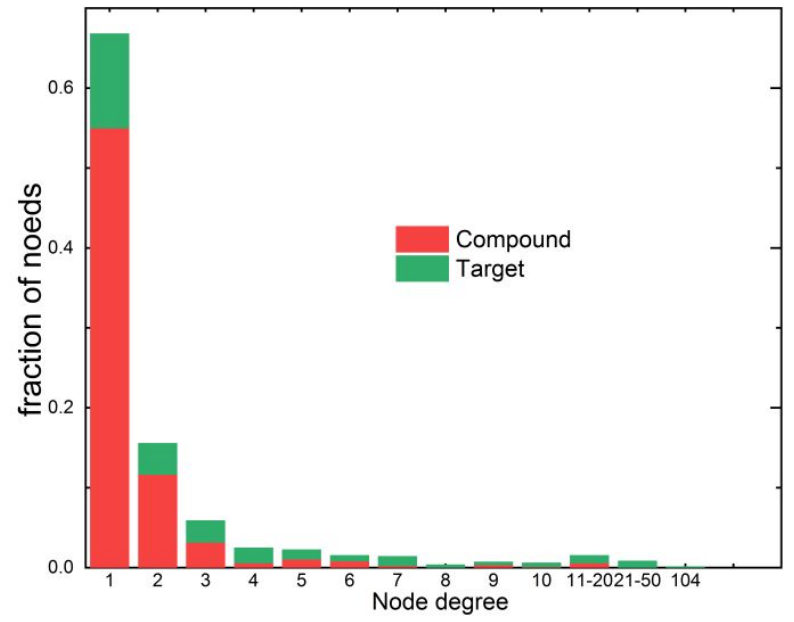

Figure S5. Node degree distribution of the compound-target network. It is approximate to power exponential distribution. 

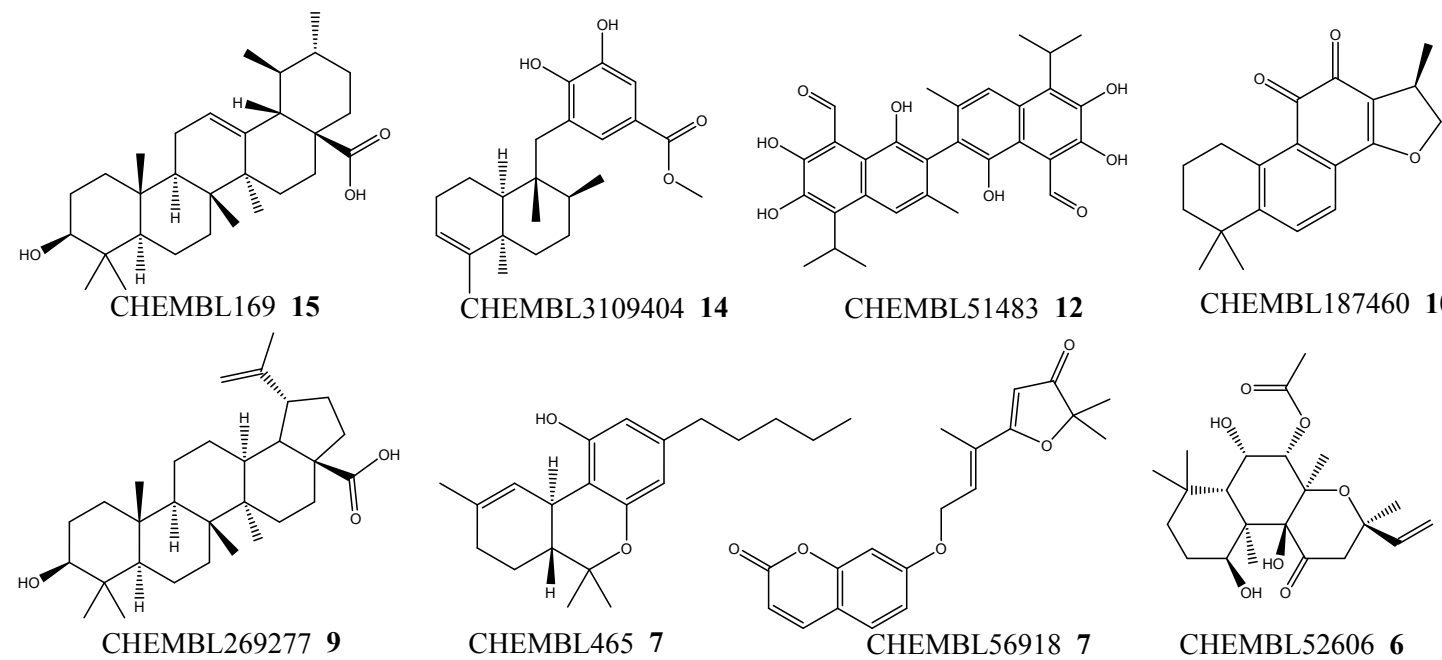

CHEMBL187460 10

CHEMBL269277 9

CHEMBL465 7

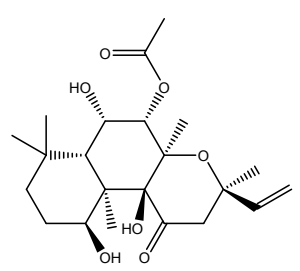

CHEMBL52606 6
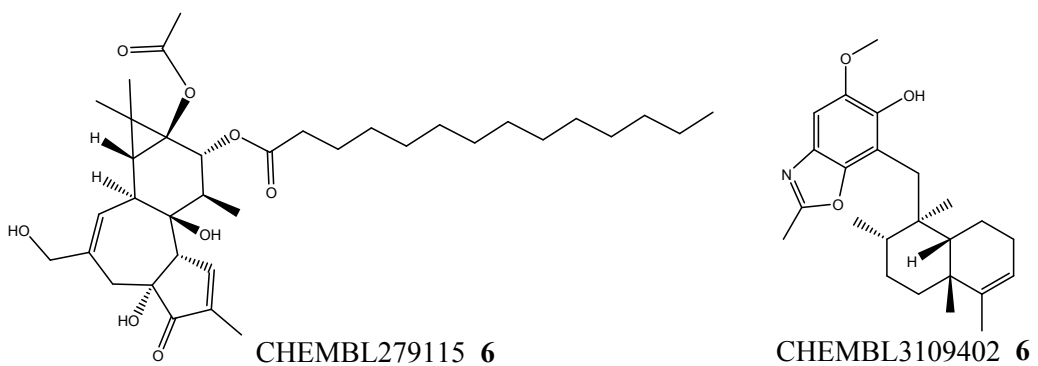

Figure S6. Top 10 active compounds, the number behind compound name means the number of targets that the active compounds act on. 

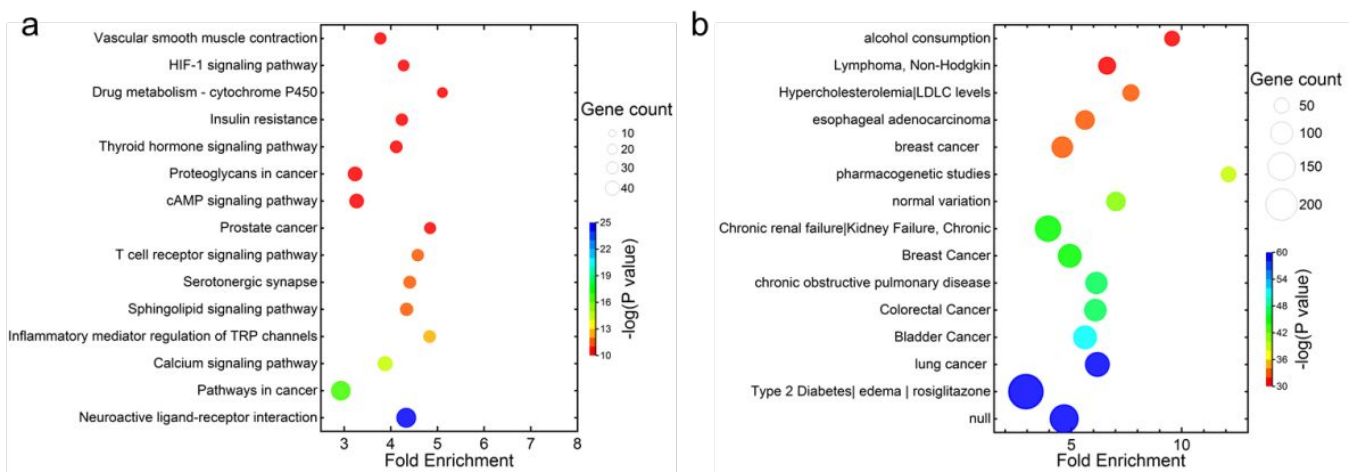

Figure S7. Top 15 enriched terms in KEGG pathway (a) and GAD disease (b) analysis. 